\title{
Transmission Strategy Design and Resource Allocation in D2D Multicast Cooperative Communications with SWIPT
}

\author{
Chenfan Weng, Dingcheng Yang $\mathbb{D}$, Jun Wan, Lin Xiao $\mathbb{D}$, and Chuanqi Zhu $\mathbb{1}$ \\ Information Engineering School, Nanchang University, Nanchang 330031, China \\ Correspondence should be addressed to Dingcheng Yang; ydcxuanyuan@msn.com
}

Received 24 May 2018; Revised 14 August 2018; Accepted 5 September 2018; Published 1 November 2018

Guest Editor: Panagiotis Demestichas

Copyright (c) 2018 Chenfan Weng et al. This is an open access article distributed under the Creative Commons Attribution License, which permits unrestricted use, distribution, and reproduction in any medium, provided the original work is properly cited.

\begin{abstract}
This paper proposes a new transmission strategy for device-to-device (D2D) multicast cooperative communication systems based on Simultaneous Wireless Information and Power Transfer (SWIPT) technology. The transmission block is divided into two slots. In the first slot, the source user transmits the information and energy to the help user by SWIPT. In the second slot, the help user uses the cellular spectrum and forwards the information to multiple receivers by using harvested energy. In this paper, we aim to maximize the total system rate, and to tackle the problem, we propose a two-step scheme: In the first step, the resource allocation problem is solved by linear programming. In the second step, the power-splitting coefficient value is obtained by taking the benefit of help user into account. Numerical results show that the proposed strategy not only effectively improves the overall throughput and spectrum efficiency but also motivates the cooperation.
\end{abstract}

\section{Introduction}

Wireless Power Communication (WPC), where the smart terminals can harvest the energy from the far-field RadioFrequency (RF) signals provided by energy access point, becomes a new approach to avoid replacing or recharging the batteries, reduce total cost, and prolong the lifetimes [1]. Simultaneous Wireless Information and Power Transfer (SWIPT) is a kind of WPC, where wireless devices can collect energy and receive information from RF signals at the same time [2-5]. In [6], Ioannis Krikidis team discussed the SWIPT technology and made a preliminary study on the allocation of wireless resources in SWIPT. Time switching and powersplitting model were proposed in [7]. Cooperative technology is also widely used in communication systems based on SWIPT. [8] studied cooperative communication system with Decode-and-Forward (DF) mode.

With the help of the fast growth of wireless communication technology, smart devices can easily access the network anywhere anytime, which makes people's sharing become ubiquitous [9]. Device-to-Device (D2D) communication was proposed to exchange information directly. The technology has several benefits, such as saving resources, improving spectral efficiency, and reducing transmission delay. Based on the transmission mode, it can be divided into unicast transmission and multicast transmission. In unicast transmission mode, a transmission channel is established between the Base Station (BS) or the sending user and each requesting user. Each channel takes a different frequency band that is orthogonal to each other, presenting a waste of spectral resources to some extent. Some works such as [10] consider throughput maximization while allowing D2D communication to underlay the cellular network; the results show that the total throughput can be increased. Some other works such as [11] consider throughput maximization under the spectral efficiency and energy constraints. Only one Cellular User (CU) and a D2D pair are considered at this scheme. [12] extends it to the general situation with multiple D2D users and CUs for maximizing the overall throughput. These works either improve network throughput [10-12] or ensure the reliability of D2D communications [13-15]. The works in $[11,16]$ take these two indicators into account at the same time. [16] has proposed an algorithm to solve a Mixed Integer and Nonlinear Programming (MINLP) resource allocation problem. But the algorithm does not consider the collaboration between CUs and D2D pair. Based on [11, 16], a maximum weight bipartite matching was proposed in [12]; the system of performance of D2D access rate and the 
total throughput improved significantly. While in multicast transmission mode the BS or the sending user is transmitting through the same frequency band with each request user, saving a certain spectrum resource, however, D2D multicast will meet more challenges which are different from unicast D2D (see, e.g., $[11,12]$ ). As the number of receivers increases, the transmission rate tends to decrease. Some works have been investigated in cooperative D2D communication [1719]. Authors in [17] proposed a cooperative caching strategy to analyze the network capacity and present an architecture to improve the network capacity. In [18], the author considers cooperative D2D communication in downlink cellular networks where the D2D transmitter is equipped with an energyharvesting capability. A network model is proposed in [19], analyzing D2D communication with RF energy harvesting.

In [20], a SWIPT-based D2D cooperative network is proposed. In SWIPT-based energy-harvesting D2D underlay network [21], the problem of joint power control and spectrum resource allocation is solved. A framework for optimal resource allocation in multicast D2D communications is presented to maximize the total throughput of D2D multicast groups and CUs in [22]. In fact, cooperative communication is hard to be realized to motivate the mobile terminals, due to the lack of incentives. Therefore, in this paper, we fully consider the benefits of the help users and aim at maximizing their benefits. Different from the previous works on cooperative communication, we proposed an innovation transmission strategy and incentive mechanism via combined D2D multicast technology with SWIPT, which can effectively improve the spectrum efficiency, lower the energy consumption, reduce the communication delay, largely release the burden of the BS, and thus reduce the cost of communication. Likewise, in order to lower the burden of the back-haul link, we assume that the content is stored locally. In addition, we will focus on the analysis of the cochannel interference brought by D2D multicast transmission and study the mechanism in user's collaboration.

\section{System Model and Transmission Protocol}

As shown in Figure 1, a cellular network is modeled in this section, consisting of a BS in the cellular center, $M$ CUs (denoted as $\mathscr{C}=\left\{C_{1}, C_{2}, C_{3}, \ldots, C_{M}\right\}$ ), and a D2D multicast group. The $\mathrm{D} 2 \mathrm{D}$ multicast group is composed of a D2D multicast source user (denoted as $D_{S}$ ), a D2D multicast help user (denoted as $D_{T}$ ), and $K$ D2D multicast receivers (denoted as $D_{R}^{k}$ ), and we define the set $\mathscr{K} \triangleq$ $D_{R}^{1}, D_{R}^{2}, \ldots, D_{R}^{K}$. We assume that D2D multicast transmission needs to be assisted by $D_{T}$ because there is no direct link due to some uncertain factors between $D_{S}$ and $D_{R}^{K}$. Therefore, this paper considers $D_{T}$ between $D_{S}$ and $D_{R}^{K}$ to cooperate the transmission. On the other hand, in a cellular network, since help user is always selfish, $D_{T}$ does not want to cooperate with $D_{S}$ by using his own energy. For this reason, we consider that $D_{T}$ collects energy and data from $D_{S}$ by SWIPT and then complete D2D multicast by using the harvested energy. And $D_{T}$ can store the excess energy in a rechargeable battery for his own use. The antenna equipped at $D_{S}$ has the function

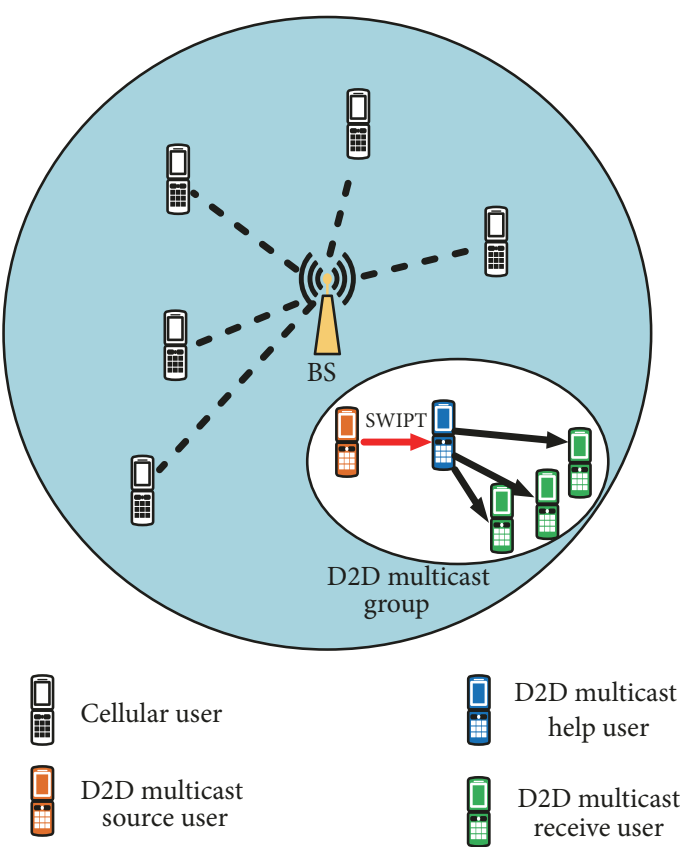

FIgURE 1: System model.

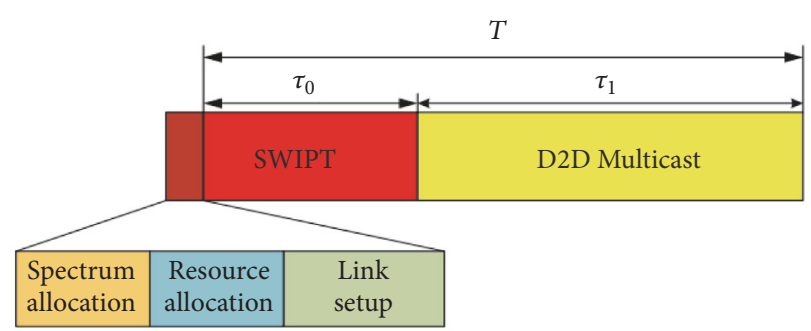

FIGURE 2: Transport protocol of WPC D2D network.

of transmitting energy and transmitting data simultaneously. $D_{T}$ is equipped with batteries, and its antenna has the function of harvesting energy. Members distributed evenly and closely in the group have the same interest. Considering there are $M$ CUs and $M$ orthogonal channels, each occupied by one CU, which are denoted as sets $\mathscr{R}=\left\{R_{1}, R_{2}, R_{3}, \ldots, R_{m}\right\}$, in this paper, we consider uplink resource sharing since reusing downlink resources will greatly reduce the spectrum efficiency according to [23].

We assume that all channels are quasistatic channels, that is, the channel coefficients remain constant for a period of transmission time. It is assumed that the channel between all users contains three kinds of loss: small-scale Rayleigh fading, the distance-dependent path loss, and long-term shadowing with loss exponent $\alpha \geq 2$. Here, let $r_{S, T}$ be the distance between $D_{S}$ and $D_{T}$ and $h_{S, T}$ be the channel coefficients between the two channels; similarly, let $r_{T, k}$ be the distance between $D_{T}$ and $K$ th $D_{R}^{k}$ and $h_{T, k}$ be the channel coefficients between the two channels.

In order to accomplish $D_{R}^{k}$, s downlink data transmission, this paper proposes a transmission strategy as shown in Figure 2. 


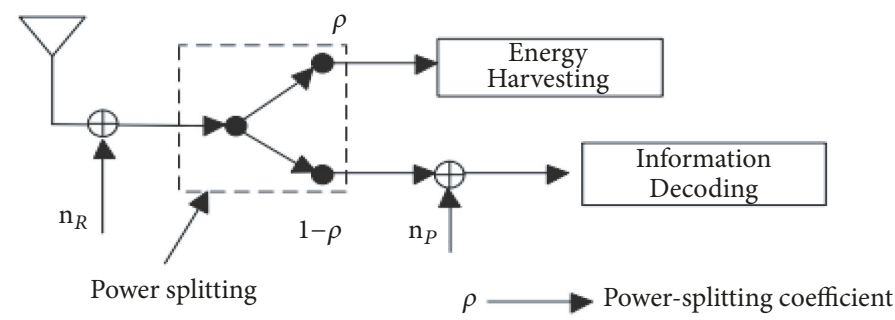

FIGURE 3: Receiving antenna of multicast help user.

The transmission block can be divided into 3 slots, which contains control signaling exchange process, SWIPT, and D2D multicast. In the first slot, the signaling exchange process consists of spectrum allocation, resource allocation, and link setup, which will be introduced in detail in Section 3. Suppose that the amount of time in the first slot is too small and therefore it can be negligible.

We assume that the total time resource meets the following constraints:

$$
\tau_{0}+\tau_{1} \leq T
$$

Without loss of generality, let $T=1$ for calculation convenience.

In the second slot, also known as SWIPT stage, $D_{S}$ transmits RF signals to $D_{T}$ with a transmit power $P_{0}$. Since SWIPT is prone to generating cochannel interference, it is assumed that $D_{S}$ transmits information and power simultaneously by the dedicated channel. Set the $D_{T}$ receiver to work in power-splitting (PS) mode. The RF signals have two uses in $D_{T}$ receiver: one is energy harvesting, and the other is information decoding, as shown in Figure 3.

The received signal at $y_{T}$ by $D_{T}$ can be expressed as

$$
y_{T}=\sqrt{P_{0} h_{S, T}} X_{i}+n,
$$

where $x_{i}$ denotes the signal that $D_{S}$ transmitted and $n$ represents the additive Gaussian noise of the antenna that complies with the form of $n \sim \mathscr{C} \mathcal{N}\left(0, \sigma^{2}\right)$. Assume that all the receiver noise satisfies this formula in this paper.

Denote the power split factor as $\rho$ with $0 \leq \rho \leq 1$, and the energy $E_{T}$ harvested by $D_{T}$ is expressed as

$$
E_{T}=\rho \eta_{T} \tau_{0} P_{0} h_{S, T}
$$

where $\eta_{T}$ is the energy conversion efficiency of $D_{T}$. Because the interference noise takes a tiny proportion in the received signal, the received noise is ignored when considering the collected energy.

In information decoding, $1-\rho$ is the information split factor of $D_{T}$, so the signal receiving rate $R_{S, T}$ is expressed as

$$
R_{S, T}=\tau_{0} \log _{2}\left(1+\frac{(1-\rho) P_{0} h_{S, T}}{\sigma^{2}}\right) .
$$

In the stage of $D 2 D$ multicast, or the $\tau_{1}$ stage, $D_{T}$ forwards data to all $D_{R}^{k}$ in the group with the energy received by the SWIPT stage by the transmitting power of $P_{T}$.
According to the energy constraint, the energy consumption of $D_{T}$ during multicast communication must be less than or equal to the energy harvested at the stage $\tau_{0}$, so we must meet the following constraints:

$$
P_{T} \tau_{1} \leq E_{T}
$$

Considering the need to reuse the CU channel in the D2D multicast transmission, $\delta_{i}(i \in \mathscr{R})$ is assumed to be a binary variable. Let $\delta_{i}=1$ be that the D2D multicast transmission is using the cellular channel $R_{i}$; then, $\delta_{i}=0$ indicates the opposite.

In the process of $\mathrm{D} 2 \mathrm{D}$ multicast communication, there are two kinds of interference in the system: (1) the cochannel interference to the $\mathrm{BS}$ when receiving the signals sent by the CUs and (2) the cochannel interference brought by $C_{m}$ to $D_{R}^{k}$ when receiving the multicast signals.

Suppose that D2D multicast communication can reuse $\mathrm{Z}$ cellular channels at most, that is,

$$
\sum^{M} \delta_{i} \leq Z, \quad i \in R
$$

In order to avoid the mass $\mathrm{CU}$ interference caused by D2D multicast communication, this paper only considers the situation of $Z=1$; that is, D2D multicast can only choose one cellular channel to communicate.

Set $\beta_{k, m}$ as a channel quality coefficient of $D_{R}^{k}$ when occupying channel $R_{m}$, and

$$
\beta_{k, m}=\frac{h_{T, k}}{\sigma^{2}+P_{m} h_{m, k}}, \quad \forall k \in K, m \in C,
$$

where $P_{m}$ is the uplink transmission power of $C_{m}$ and $h_{m, k}$ is the channel coefficient between $C_{m}$ and $D_{R}^{k}$.

In D2D multicast group, due to different channel coefficients of $D_{R}^{k}$ and $D_{T}$, each $D_{R}^{k}$ receives a different rate during multicast transmission. In order to ensure that each $D_{R}^{k}$ can complete multicast communication, we define the multicast channel quality coefficient $\beta_{m}^{D}$ as that of the receiver with the worst channel quality in the group related to $D_{T}$, which will meet the following:

$$
\beta_{m}^{D}=\min \beta_{k, m}, \quad \forall k \in K
$$


Therefore, the normalized rate $R_{T, R}$ of multicast $\mathrm{D} 2 \mathrm{D}$ group can be written as follows:

$$
R_{T, R}=\sum_{m-1}^{M} \delta_{m} \tau_{1} \log _{2}\left(1+P_{T} \beta_{m}^{D}\right) .
$$

Meanwhile, it should also meet the information constraints in stage $\tau_{1}$ :

$$
R_{T, R} \tau_{1} \leq R_{p, T} \tau_{0}
$$

The multicast communication rate $R_{T, R}$ of D2D multicast group meets the following constraints:

$$
R_{T, R}^{\text {sum }} \leq \sum_{m-1}^{M} \delta_{m} K \tau_{1} \log _{2}\left(1+P_{T} \beta_{m}^{D}\right)
$$

For CUs, let $r_{p, m}$ and $h_{p, m}$ denoted the distance and the channel coefficient between $C_{m}$ and BS respectively, $r_{T, p}$ and $h_{T, p}$ denoted the distance and the channel coefficient between $\mathrm{BS}$ and $D_{T}$ respectively, then the channel quality coefficient between $C_{m}$ and BS can be formulated as follows:

$$
\beta_{m}=\frac{h_{p, m}}{\sigma^{2}+\delta_{m} P_{T} h_{T, p}}
$$

Assuming that the transmission power $C_{m}$ remains unchanged during the whole transmission process $\mathrm{T}$, then the transmission rate is

$$
R_{m}=\log _{2}\left(1+P_{m} \beta_{m}\right)
$$

In order to ensure the service quality of CUs and D2D during the communication, the following constraints should be met:

$$
\begin{aligned}
R_{T, R} & \geq R_{\text {min }}^{C U} . \\
R_{m} & \geq R_{\text {min }}, \quad \forall m \in M
\end{aligned}
$$

Table 1 lists the variables and parameters used in the paper.

\section{Problem Description and Optimization}

In this paper, we endeavor to maximize the total system rate by combining the optimization of frequency resources, power split factors, and transmission power in the case of satisfying the constraints mentioned above. Thus, the optimization problem can be formulated as follows:
TABLE 1: Table of notations.

\begin{tabular}{lc}
\hline Notation & Description \\
\hline $\mathscr{C}$ & Set of cellular users (CU) \\
$\mathscr{K}$ & Set of D2D multicast receivers \\
$\mathscr{R}$ & Set of orthogonal channels \\
$D_{S}$ & D2D multicast source user \\
$D_{T}$ & D2D multicast help user \\
$D_{R}^{k}$ & D2D multicast receivers \\
$r_{S, T}$ & The distance between $D_{S}$ and $D_{T}$ \\
$h_{S, T}$ & The channel coefficients between the two channels \\
$r_{T, k}$ & The distance between $D_{T}$ and Kth $D_{R}^{k}$ \\
$h_{T, k}$ & The power split factor \\
$\rho$ & The energy harvested by $D_{T}$ \\
$E_{T}$ & The energy conversion efficiency of $D_{T}$ \\
$\eta_{T}$ & The signal receiving rate \\
$R_{S, T}$ & The transmitting power \\
$P_{T}$ & A binary variable \\
$\delta_{i}$ & The normalized rate of multicast $\mathrm{D} 2 \mathrm{D}$ group \\
$\beta_{k, m}$ & The summation of normalized rate \\
$P_{m}$ & The channel coefficient between $C_{m}$ and $D_{R}^{k}$ \\
$h_{m, k}$ & Multicast channel quality coefficient \\
$\rho_{m}^{D}$ & The \\
$R_{T, R}$ & $R_{T, R}^{s u m}$
\end{tabular}

$$
\begin{aligned}
\text { (P1) } \underset{\delta_{m}, \rho, P_{T}, P_{m}}{\operatorname{maxmize}} & \left(R_{T, R}+\sum_{m-1}^{M} R_{m}\right) \\
\text { s.t. } \quad & P_{T} \tau_{1} \leq E_{T} \\
& \sum^{M} \delta_{i} \leq 1, \quad i \in R \\
& R_{T, R} \tau_{1} \leq R_{S, T} \tau_{0} \\
& R_{T, R} \geq R_{\min }^{C U} \\
& R_{m} \geq R_{\min }^{C U}, \quad \forall m \in M \\
& \delta_{m} \in\{0,1\} \\
& \beta_{m}^{D 2 D}=\min \beta_{k, m}, \quad \forall k \in K \\
& 0 \leq P_{T} \leq P_{T}^{\max } \\
& 0 \leq P_{m} \leq P_{m}^{\max } .
\end{aligned}
$$

In general, MINLP is NP-hard problem, but in this paper, we consider the problem of MINLP for the special case (each D2D group can reuse the channels of at most one CU and each CU can share their channels with at most one D2D group); it is a bipartite problem.

The algorithm proposed in (16) will be divided into three steps: first, fix the value of $\delta_{i}$; then optimize the value of each $\delta_{i}$, and if the optimization is not feasible, the channel will be eliminated; finally, the channel that has the best effect in the remaining feasible solution is selected as the reusable channel. 
When $\delta_{i}$ is fixed, that is,

$$
\delta_{i}= \begin{cases}1 & i=m \\ 0 & \text { else }\end{cases}
$$

with only one cellular channel $R_{m}$ being reused, we just take the rate of the CUs in the reused band among the targeted users that are being optimized.

For the constrained condition (8), to obtain $\beta_{m}^{D}$, we have to find out the channel quality coefficient of the receiver with the worst channel quality and compare the interference values between all $D_{R}^{k}$. When considering the reuse of a cellular channel, formula (7) shows that, for each $D_{R}^{k}$ under the same $P_{m}, \beta_{k, m}$ is only associated with $h_{m, k}$ and $h_{T, k}$. The smaller $h_{T, k}$ and the larger $h_{m, k}$ become, the smaller the value of $\beta_{k, m}$ will be. To conclude, we define $\gamma_{k}=h_{T, k} / h_{m, k}$ which means when $\gamma_{k}$ picks the minimum value, the receiving user $\beta_{k^{*}, m}$ goes to $\beta_{m}^{D}$ follows:

The original optimization problem (16) is simplified as

$$
\text { (P2) } \max _{\rho_{m}, P_{T}, P_{m}} \quad \begin{gathered}
\tau_{1} \log _{2}\left(1+\frac{P_{T} h_{T, k^{*}}}{\sigma^{2}+P_{m} h_{m, k^{*}}}\right) \\
\quad+\log _{2}\left(1+\frac{P_{m} h_{p, m}}{\sigma^{2}+P_{T} h_{T, p}}\right) \\
\text { s.t. } \quad P_{T} \tau_{1} \leq \rho_{m} \eta_{T} \tau_{0} P_{0} h_{S, T} \\
\quad \tau_{1} \log _{2}\left(1+\frac{P_{T} h_{T, k^{*}}}{\sigma^{2}+P_{m} h_{m, k^{*}}}\right) \\
\leq \tau_{0} \log _{2}\left(1+\frac{\left(1-\rho_{m}\right) P_{0} h_{S, T}}{\sigma^{2}}\right) \\
\tau_{1} \log _{2}\left(1+\frac{P_{T} h_{T, k^{*}}}{\sigma^{2}+P_{m} h_{m, k^{*}}}\right) \geq R_{\min }^{D 2 D} \\
\log _{2}\left(1+\frac{P_{m} h_{p, m}}{\sigma^{2}+P_{T} h_{T, p}}\right) \geq R_{\min }^{C U} \\
0 \leq P_{T} \leq P_{T}^{\max }, 0 \leq P_{m} \leq P_{m}^{\max } \\
k^{*}=\arg \min _{k} \gamma_{k} .
\end{gathered}
$$

From the objective function expression and the constraints, the problem is still a nonconvex problem and cannot be solved by the traditional convex optimization method. Therefore, in this paper, we are going to divide the problem into two subproblems.

3.1. Optimization Algorithm for D2D Interference. Firstly, the problem of D2D interference is to be optimized. Problem (18) simplifies subproblem (19):

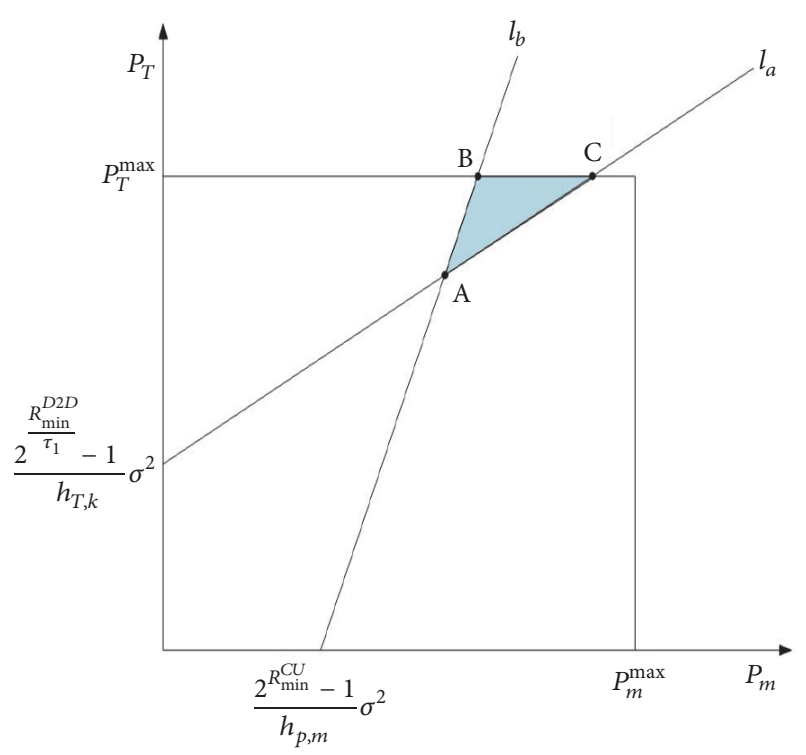

FIgURE 4: Case 1.

$$
\begin{aligned}
& \text { (P3) } \max _{P_{T}, P_{m}} \tau_{1} \log _{2}\left(1+\frac{P_{T} h_{T, k^{*}}}{\sigma^{2}+P_{m} h_{m, k^{*}}}\right) \\
&+\log _{2}\left(1+\frac{P_{m} h_{p, m}}{\sigma^{2}+P_{T} h_{T, p}}\right) \\
& \text { s.t. } \quad \mathbf{C}_{\mathbf{a} 1}: \tau_{1} \log _{2}\left(1+\frac{P_{T} h_{T, k^{*}}}{\sigma^{2}+P_{m} h_{m, k^{*}}}\right) \\
& \geq R_{\text {min }}^{D 2 D} \\
& \quad \mathbf{C}_{\mathbf{a} 2}: \log _{2}\left(1+\frac{P_{m} h_{p, m}}{\sigma^{2}+P_{T} h_{T, p}}\right) \geq R_{\text {min }}^{C U} \\
& \mathbf{C}_{\mathbf{a} 3}: 0 \leq P_{T} \leq P_{T}^{\max } \\
& \mathbf{C}_{\mathbf{a} 4}: 0 \leq P_{m} \leq P_{m}^{\max } .
\end{aligned}
$$

The formulas $\left(C_{a 1}\right)$ and $\left(C_{a 2}\right)$ are simplified as

$$
\begin{aligned}
& P_{T} \geq A\left(\sigma^{2}+P_{m} h_{m, k^{*}}\right) \\
& P_{m} \geq B\left(\sigma^{2}+P_{T} h_{T, p}\right),
\end{aligned}
$$

where $A=\left(2^{R_{\min }^{D 2 D} / \tau_{1}}-1\right) / h_{T, k^{*}}$ and $B=\left(2^{R_{\min }^{C U} / \tau_{1}}-1\right) / h_{p, m}$. Combined with $\left(C_{a 3}\right),\left(C_{a 4}\right),(20)$, and (21), it is known that the constraints are all linear, which are divided into 6 cases as shown in Figures 4-9. The line $l_{a}$ represents constraint (20) with equality, the slope is $A h_{m, k^{*}}$, and the intersection point with the $P_{T}$ axis is $\left(0, A \sigma^{2}\right)$. When constraint (20) is met, the feasible solution is in the upper side of the line $l_{a}$. The line $l_{b}$ represents constraint (21) with equality and the intersection point with the $P_{m}$ axis, and the slope is $1 / \mathrm{B}$. When constraint (21) is met, the feasible solution is on the right side of the line $l_{b}$.

In Figure 4, point $\mathrm{C}$ coordinates at $\left(\left(\left(P_{T}^{\max } / A\right)-\right.\right.$ $\left.\left.\sigma^{2}\right) / h_{m, k^{*}}, P_{T}^{\max }\right)$, and point $\mathrm{B}$ coordinates at $\left(B\left(\sigma^{2}+\right.\right.$ 


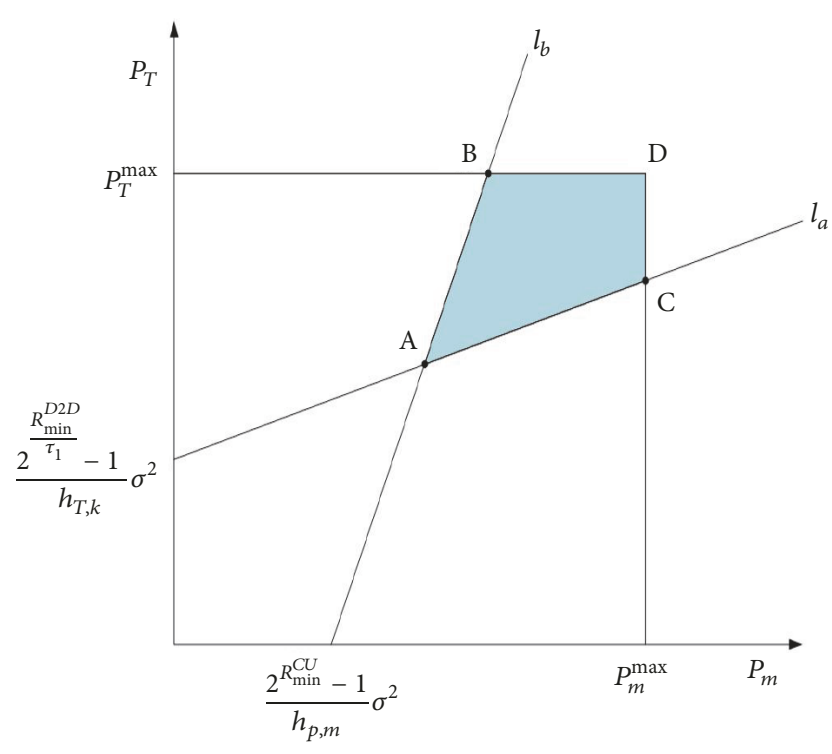

Figure 5: Case 2.

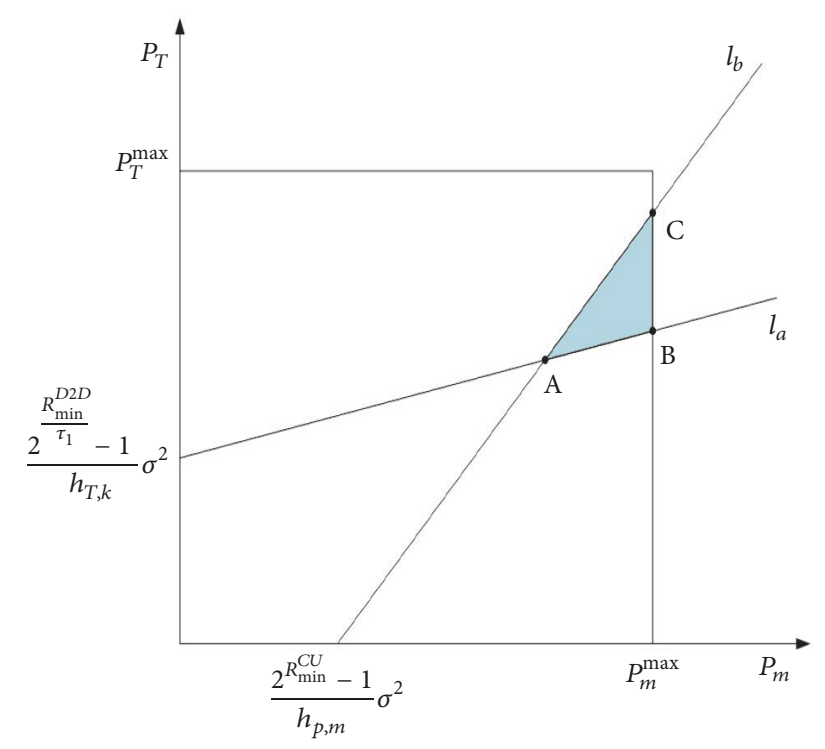

FIgURE 6: Case 3.

$\left.\left.P_{T}^{\max } h_{T, p}\right), P_{T}^{\max }\right)$. Therefore, case 1 meets the following conditions:

$$
\begin{gathered}
\frac{\left(P_{T}^{\max } / A\right)-\sigma^{2}}{h_{m, k}} \leq P_{m}^{\max } \\
B\left(\sigma^{2}+P_{T}^{\text {max }} h_{T, p}\right) \leq P_{m}^{\max } .
\end{gathered}
$$

Suppose $\widehat{P}_{T}^{*}$ and $\widehat{P}_{m}^{*}$ are the best solutions for problem (19). In Figures $4-6$, the blue part represents a feasible value region. In Figures 7-8, there is no value region that meets the constraints; in other words, there is no solution to the problem.

In Figure 5, point $\mathrm{B}$ coordinates at $\left(B\left(\sigma^{2}+\right.\right.$ $\left.\left.P_{T}^{\max } h_{T, p}\right), P_{T}^{\max }\right)$, and point $\mathrm{C}$ coordinates at $\left(P_{m}^{\max }, A\left(\sigma^{2}+\right.\right.$

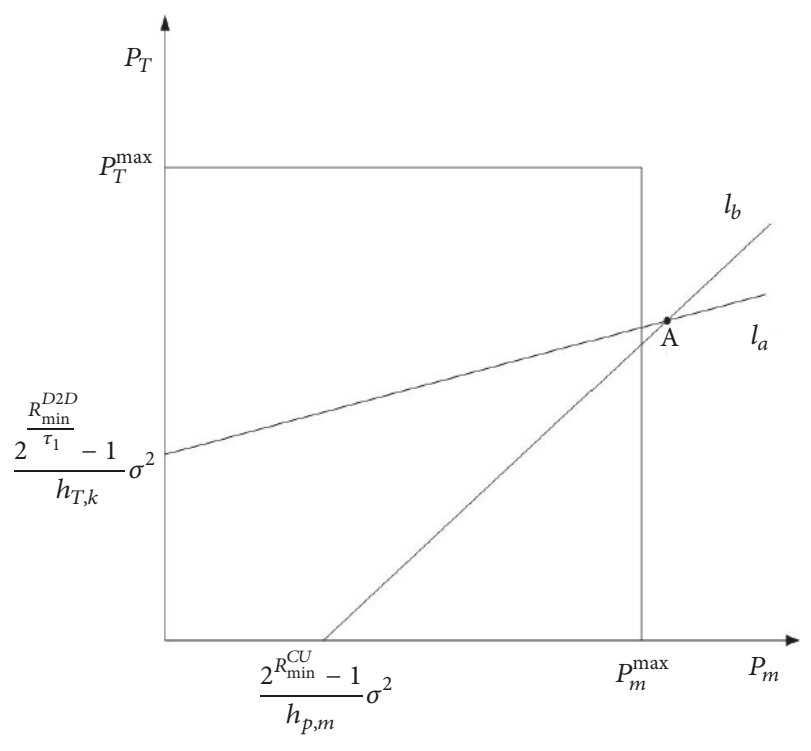

Figure 7: Case 4.

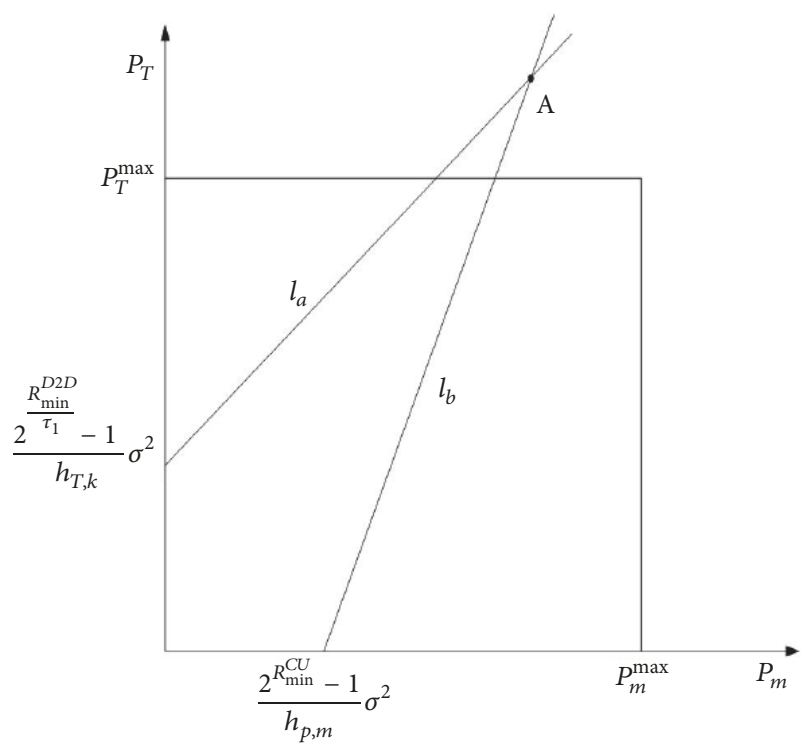

Figure 8: Case 5.

$\left.P_{m}^{\max } h_{m, k^{*}}\right)$. Therefore, case 2 meets the following constraints:

$$
\begin{gathered}
B\left(\sigma^{2}+P_{T}^{\max } h_{T, p}\right) \leq P_{m}^{\max } \\
A\left(\sigma^{2}+P_{m}^{\max } h_{m, k^{*}}\right) \leq P_{T}^{\max } .
\end{gathered}
$$

In Figure 6 , point $\mathrm{B}$ coordinates at $\left(P_{m}^{\max }, A\left(\sigma^{2}+\right.\right.$ $\left.\left.P_{m}^{\max } h_{m, k^{*}}\right)\right)$, and point $\mathrm{C}$ coordinates at $\left(P_{m}^{\max },\left(P_{m}^{\max } / B-\right.\right.$ $\left.\left.\sigma^{2}\right) / h_{T, p}\right)$. Thus, case 3 meets the following constraints:

$$
\begin{aligned}
A\left(\sigma^{2}+P_{m}^{\max } h_{m, k^{*}}\right) & \leq P_{T}^{\max } \\
\frac{\left(P_{m}^{\max } / B\right)-\sigma^{2}}{h_{T, p}} & \leq P_{T}^{\max } .
\end{aligned}
$$




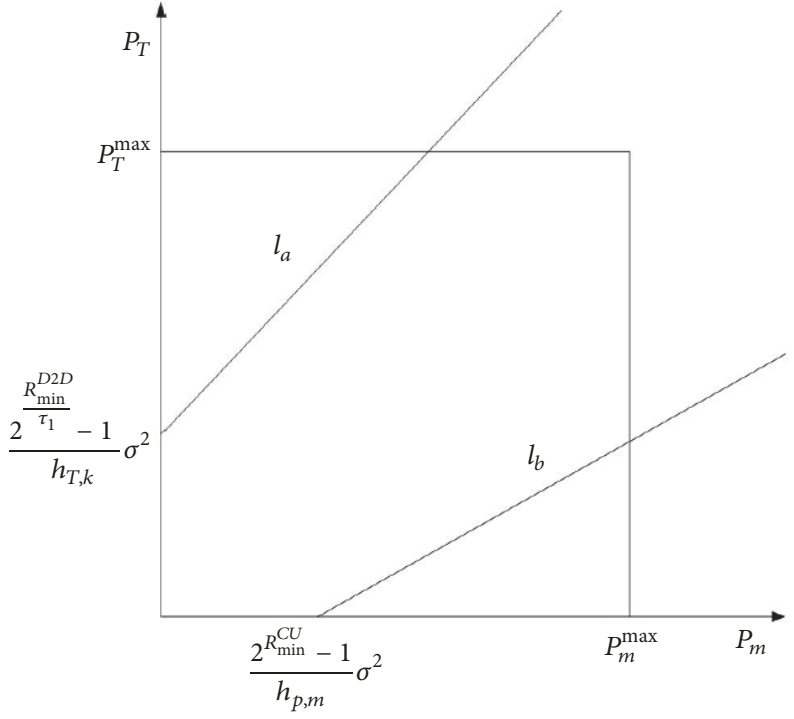

Figure 9: Case 6.

All the situations from Figures 7-9 do not meet the requirements ranging from (22) to (24), and it is known from the function curve (19) that there is no feasible solution.

Next, we analyze the objective function (19). According to [24], we can prove that when a set of solutions $\left(P_{m}, P_{T}\right)$ is taken within the feasible solution region, another set of solutions $\left(\mu P_{m}, \mu P_{T}\right)$ will be found in the feasible region and meets the condition of $f\left(\mu P_{m}, \mu P_{T}\right) \geq f\left(P_{m}, P_{T}\right)$, in which

$$
\begin{aligned}
f\left(P_{m}, P_{T}\right) \triangleq & \tau_{1} \log _{2}\left(1+\frac{P_{T} h_{T, k^{*}}}{\sigma^{2}+P_{m} h_{m, k^{*}}}\right) \\
& +\log _{2}\left(1+\frac{P_{m} h_{p, m}}{\sigma^{2}+P_{T} h_{T, p}}\right) .
\end{aligned}
$$

Therefore, at least one of the optimal solutions $\widehat{P}_{T}^{*}$ and $\widehat{P}_{m}^{*}$ of problem (19) can take the maximum value; that is, constraints in $\left(C_{a 3}\right)$ or $\left(C_{a 4}\right)$ can be set equal.

(i) For case 1 , the optimal solution $\left(\widehat{P}_{T}^{*}, \widehat{P}_{m}^{*}\right)$ lies in the line BC; then $\widehat{P}_{T}^{*}=P_{T}^{\max }$. When $P_{T}$ is fixed, the objective function (19) is a convex function, so the optimal solution $\left(\widehat{P}_{T}^{*}, \widehat{P}_{m}^{*}\right)$ can only be point $\mathrm{B}$ or point $\mathrm{C}$.

(ii) For case 2, the optimal solution $\left(\widehat{P}_{T}^{*}, \widehat{P}_{m}^{*}\right)$ may be in line $\mathrm{BD}$ or $\mathrm{CD}$, and similar to case 1 , the optimal solutions only may be $\mathrm{B}, \mathrm{C}$ or $\mathrm{D}$.

(iii) For case 3 , the optimal solution $\left(\widehat{P}_{T}^{*}, \widehat{P}_{m}^{*}\right)$ lies in the segment $\mathrm{BC}$, and similar to case 1 , the optimal solution is only possible for $\mathrm{B}$ or $\mathrm{C}$.

In summary, we can find that the optimal solution of subproblem (16) is as follows.

$$
\begin{aligned}
& \text { When }\left(\left(P_{T}^{\max } / A\right)-\sigma^{2}\right) / h_{m, k^{*}} \leq P_{m}^{\max } \text { and } B\left(\sigma^{2}+\right. \\
& \left.P_{T}^{\max } h_{T, p}\right) \leq P_{m}^{\max }, \\
& \left(\widehat{P}_{T}^{*}, \widehat{P}_{m}^{*}\right) \\
& =\arg \max \left\{\begin{array}{l}
f\left(\frac{\left(P_{T}^{\max } / A\right)-\sigma^{2}}{h_{m, k^{*}}}, P_{T}^{\max }\right), \\
f\left(B\left(\sigma^{2}+P_{T}^{\max } h_{T, p}\right), P_{T}^{\max }\right)
\end{array}\right\} .
\end{aligned}
$$
$P_{T}^{\max }$,

When $B\left(\sigma^{2}+P_{T}^{\max } h_{T, p}\right) \leq P_{m}^{\max }$ and $A\left(\sigma^{2}+P_{m}^{\max } h_{m, k^{*}}\right) \leq$

$$
\begin{aligned}
& \left(\widehat{P}_{T}^{*}, \widehat{P}_{m}^{*}\right) \\
& =\arg \max \left\{\begin{array}{c}
f\left(B\left(\sigma^{2}+P_{T}^{\text {max }} h_{T, p}\right), P_{T}^{\text {max }}\right), \\
f\left(P_{m}^{\text {max }}, A\left(\sigma^{2}+P_{m}^{\text {max }} h_{m, k^{*}}\right)\right), \\
f\left(P_{m}^{\max }, P_{T}^{\text {max }}\right)
\end{array}\right\} .
\end{aligned}
$$
$P_{T}^{\max }$,

$$
\begin{aligned}
& \left(\widehat{P}_{T}^{*}, \widehat{P}_{m}^{*}\right) \\
& \quad=\arg \max \left\{\begin{array}{c}
f\left(P_{m}^{\max }, A\left(\sigma^{2}+P_{m}^{\text {max }} h_{m, k^{*}}\right)\right), \\
f\left(P_{m}^{\max }, \frac{P_{m}^{\max } / B-\sigma^{2}}{h_{T, p}}\right)
\end{array}\right\} .
\end{aligned}
$$

When the abovementioned three conditions are not met, there is no solution to the original problem.

3.2. Joint Power Split Factor and D2D Interference Optimization Algorithm. According to the optimization results of Section 3.1, this section will optimize the power split factor $\rho$; then, the optimization problem will become

$$
\begin{array}{ll}
\text { (P4) Find } & \rho_{m} \\
\text { s.t. } & \widehat{P}_{T}^{*} \tau_{1} \leq \rho_{m} \eta_{T} \tau_{0} P_{0} h_{S, T} \\
& \tau_{1} \log _{2}\left(1+\frac{\widehat{P}_{T}^{*} h_{T, k^{*}}}{\sigma^{2}+\widehat{P}_{m}^{*} h_{m, k^{*}}}\right) \\
& \leq \tau_{0} \log _{2}\left(1+\frac{\left(1-\rho_{m}\right) P_{0} h_{S, T}}{\sigma^{2}}\right) .
\end{array}
$$

The following constraints can be derived:

$$
\begin{aligned}
& \frac{\widehat{P}_{T}^{*}}{\eta_{T} \tau_{0} P_{0} h_{S, T}} \leq \rho_{m} \\
& \quad \leq 1-\frac{\sigma^{2}}{P_{0} h_{S, T}}\left[2^{\tau_{1}-\tau_{0}}\left(1+\frac{\widehat{P}_{T}^{*} h_{T, k^{*}}}{\sigma^{2}+\widehat{P}_{m}^{*} h_{m, k^{*}}}\right)-1\right] .
\end{aligned}
$$

When $\rho_{m}$ meets constrained condition (30), the original problem (18) has feasible solutions.

In order to encourage $D_{T}$ to carry out cooperative communication and ensure the best benefits of its users, it should 
get the maximum energy in the process of cooperation, so let the energy harvested be $\triangle$, that is,

$$
\triangle=\rho_{m} \eta_{T} \tau_{0} P_{0} h_{S, T}-\widehat{P}_{T}^{*} \tau_{1}
$$

When $\rho_{m}$ is maximized, $\triangle$ goes to the maximum, so set

$$
\rho_{m}^{*}=1-\frac{\sigma^{2}}{P_{0} h_{S, T}}\left[2^{\tau_{1}-\tau_{0}}\left(1+\frac{\widehat{P}_{T}^{*} h_{T, k^{*}}}{\sigma^{2}+\widehat{P}_{m}^{*} h_{m, k^{*}}}\right)-1\right]
$$

Thus, problem (18) is solved.

Next, let $i=m+1$, then, changing the value of $\delta_{m}$ and continuing to solve problem (18).

After traversing all channels, find out the best reuse cellular channel $m^{*}=\arg \max R_{\text {sum }}^{m}$ and output the global optimal solution.

Hence, original problem (16) is solved.

To conclude, the algorithms proposed in this paper are summarized as shown in Algorithm 1.

\section{Simulation Result}

Figure 10 simulates a wireless cellular D2D cooperative communication system with a size of $550 * 500 \mathrm{~m}^{2}$ with the BS located at $(0,0)$. The abscissas of $D_{S}, D_{T}$, and $D_{R}^{k}$ are 500 . The coordinates of several CUs are between the BS and D2D users. The number of multicast transmission receiving users is 5 , and all the users are within the radius of $10 \mathrm{~m}$. Set both the maximum transmission power $P_{T}^{\max }$ and $P_{m}^{\max }$ of the user as $24 \mathrm{dBm}$ and $\tau_{0}$ and $\tau_{1}$ as 0.5 . Assume the value of $R_{\min }^{D 2 D}$ and $R_{\text {min }}^{C U}$ obeys the uniform distribution of $[0,5](\mathrm{bps} / \mathrm{Hz})$. All channels meet $h_{i j}=\beta_{i j} \theta_{i j} r_{i j}^{-\alpha}$, in which $\beta_{i j}$ is large-scale fading and $\theta_{i j}$ is small-scale fading. The noise of all the received antennas is $-114 \mathrm{dbm}$.

Figure 11 is a simulation diagram under the scenario where the number of CUs is 15 when $\alpha=3$. As shown in the figure, the blue baseline, about $9.301 \mathrm{bps} / \mathrm{Hz}$, is the rate of CUs when there is no interference from D2D multicast communication. When the average distance between $D_{T}$ and $D_{R}^{k}$ increases, due to $\mathrm{D} 2 \mathrm{D}$ cochannel interference, the rate of CUs is slightly lower than the baseline but generally remains the same. In this band, the rate of D2D multicast users can be greatly improved, remarkably increasing the spectrum utilization rate of the system. What is more, because of the influence of propagation path loss, the propagation rate of multicast is reduced when the average distance between $D_{T}$ and $D_{R}^{k}$ increases, but a certain communication rate can be still guaranteed.

The simulation scenario in Figure 12 shows the total system rate when the distance between $D_{T}$ is $50 \mathrm{~m}$. It can be drawn that when the number of CUs increases, D2D multicast communication will have more probability to reuse cellular channels with smaller cochannel interference, so the system rate will rise obviously at the beginning. But when the number of CUs rises to around 40, the system tends to a stable value. At this point, D2D multicast communication can select a better channel transmission from 40 CUs, ensuring that the system has a large total transmission rate. Therefore,

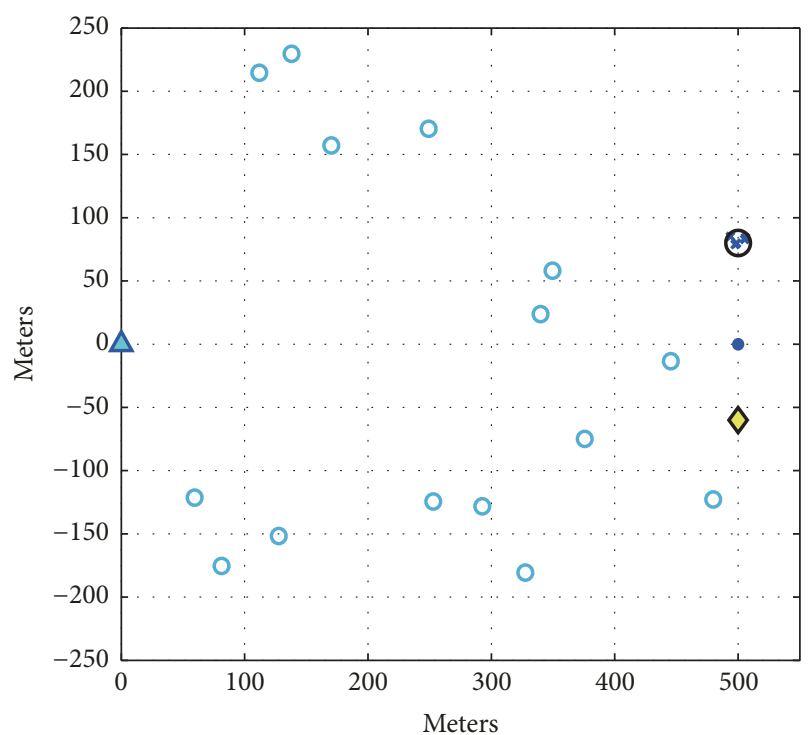

$\begin{array}{ll}\triangle & \text { Base station } \\ * & \text { D2D multicast receive user } \\ \text { - D2D multicast help user } \\ \diamond \text { D2D multicast source user } \\ \diamond \text { Cellular user }\end{array}$

FIGURE 10: Wireless cellular D2D cooperative communication system.

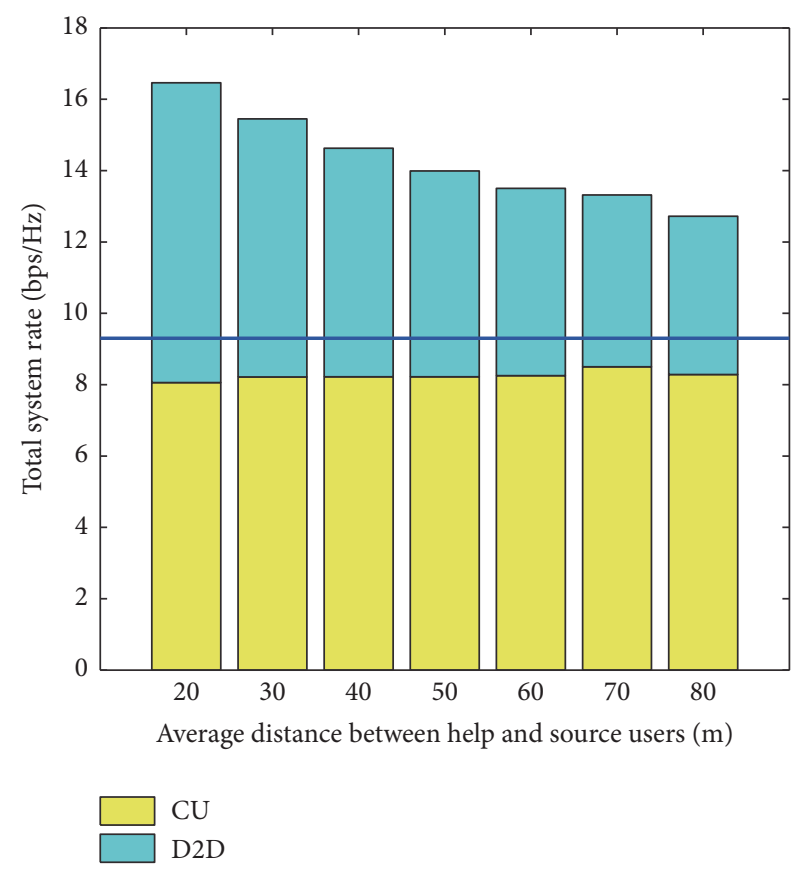

FIGURE 11: System transmission rate (a).

without the occurrence of network congestion, the number of the best CUs in the communication system in this area is 40. In addition, it can be seen from the figure that the change of the channel coefficient will significantly affect the total rate of the system. 


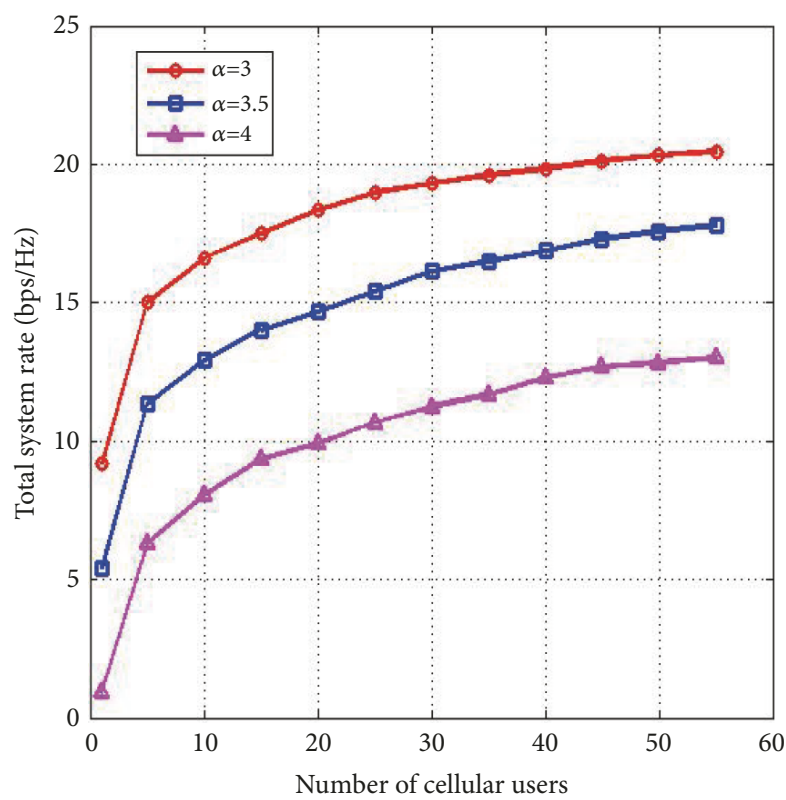

FIGURE 12: System transmission rate (b).

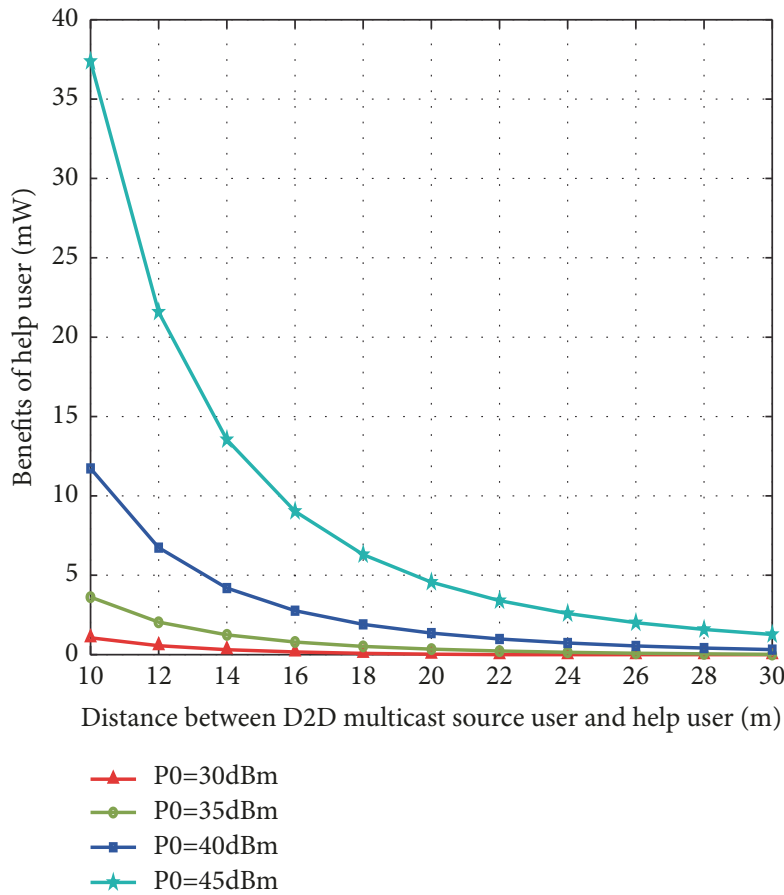

FIGURE 13: Energy benefits of collaborative users.

Figure 13 is a help user benefit with a distance between $D_{T}$ and $D_{R}^{k}$ of $50 \mathrm{~m}$ and a number of 15 CUs when $\alpha=$ 3. It can be seen from the graph that when the distance between $D_{S}$ and $D_{R}^{k}$ increases, the energy harvested by ${ }_{T}$ will be greatly reduced due to the influence of path loss, resulting in its lower revenue. And Figure 13 shows that when $P_{0}$ is set as $30 \mathrm{dBm}-40 \mathrm{dBm}$, and the distance between $D_{S}$ and $D_{T}$ is too long, the energy revenue is reduced to 0 , and at this point $D_{T}$ is very likely to consume its own
1. Initialize $m=1$.
2. If $m<M$, order $\delta_{i}=\left\{\begin{array}{ll}1 & i=m \\ 0 & \text { else }\end{array}\right.$, if not, jump to step 5.

Solve problem(19):

When the restricted condition (22) is satisfied, the optimal solution $\left(\widehat{P}_{T}^{*}, \widehat{P}_{m}^{*}\right)$ is equation (26); When the restricted condition (23) is satisfied, the optimal solution $\left(\widehat{P}_{T}^{*}, \widehat{P}_{m}^{*}\right)$ is equation (27); When the restricted condition (24) is satisfied, the optimal solution $\left(\widehat{P}_{T}^{*}, \widehat{P}_{m}^{*}\right)$ is equation (28);

If the above restricted conditions are not satisfied, output the optimal solution $\mathbf{O}_{\mathrm{m}}=\emptyset$.

3.If the solution of step 2 satisfies the condition (30), output $\rho_{m}^{*}$ is equation (32). Or else, $\mathbf{O}_{\mathbf{m}}=\emptyset$.

4.Here, the optimal solution of problem (18) is an array $\mathbf{O}_{\mathbf{m}}=\left(\widehat{P}_{T}^{*}, \widehat{P}_{m}^{*}, \rho_{m}^{*}\right)$, calculate $R_{\text {sum }}^{m}$ by formula (19).

Update $m=m+1$, return step 2 .

5.Find the best channel $m^{*}=\arg \max R_{\text {sum }}^{m}$,

and output the optimal solution $O_{m}^{*}=O_{m}$.

Algorithm 1: Joint Power Split Factor and D2D Interference Optimization Algorithm.

energy for cooperative communication, thus reducing the enthusiasm of the user's collaboration. Therefore, when we find that the distance between $D_{S}$ and $D_{T}$ is too far, we should increase transmission power and ensure $D_{T}$ 's cooperative revenue.

\section{Conclusion}

In this paper, SWIPT, D2D multicast technology, and user collaboration technology are combined to build up a D2D multicast cooperative communication system based on SWIPT, and a new transmission strategy is proposed. The objective of the research is to maximize the total system rate. Because of the problem of MINLP, the rate optimization problem is divided into D2D interference problem and power split factor optimization problem in this paper. To solve the D2D interference problem, we use linear programming to figure out the optimal transmission power and the optimal reusable cellular channel. In order to optimize the power split factor, we fully consider the benefits of the help users and aim at maximizing their benefits, figuring out the optimal power split factor. The simulation results show that the strategy proposed in this paper can significantly increase the total rate and spectrum utilization of the system, ensuring the benefits of the help users to a certain extent.

\section{Data Availability}

The data used to support the findings of this study are included within the article. 


\section{Conflicts of Interest}

The authors declare that there are no conflicts of interest regarding the publication of this paper.

\section{Acknowledgments}

This work was supported in part by the National Natural Science Foundation of China (61703197, 61561032, and 61461029), China/Jiangxi Postdoctoral Science Foundation Funded Projet (2014MT561879, 2014KY046), Young Scientists Project Funding of Jiangxi Province (20162BCB23010, 2015BCB23020), the Natural Science Foundation of Jiangxi Province (20114ACE00200), and Graduate Student Innovation Special Funds of Nanchang University (Grant no. CX2017190).

\section{References}

[1] Y. Shi, L. Xie, Y. T. Hou, and H. D. Sherali, "On renewable sensor networks with wireless energy transfer," in Proceedings of the IEEE (INFOCOM '11), pp. 1350-1358, Shanghai, China, April 2011.

[2] S. Bi, C. K. Ho, and R. Zhang, "Wireless powered communication: opportunities and challenges," IEEE Communications Magazine, vol. 53, no. 4, pp. 117-125, 2015.

[3] H. Chen, L. Xiao, D. Yang, T. Zhang, and L. Cuthbert, "User Cooperation in Wireless Powered Communication Networks with a Pricing Mechanism," IEEE Access, vol. 5, pp. 16895-16903, 2017.

[4] C. Zhu, D. Yang, X. Shen, L. Xiao, and L. Cuthbert, "Optimal Pricing and User Cooperation for Utility-Efficient Wireless Powered Communications," Wireless Personal Communications, vol. 96, no. 1, pp. 599-619, 2017.

[5] F. Wu, L. Xiao, D. Yang, L. Cuthbert, and X. Liu, “Transceiver designs for interference alignment based cognitive radio networks with energy harvesting," Wireless Personal Communications, vol. 98, no. 2, pp. 1895-1911, 2018.

[6] I. Krikidis, S. Timotheou, S. Nikolaou, G. Zheng, D. W. K. $\mathrm{Ng}$, and R. Schober, "Simultaneous Wireless Information and Power Transfer in modern communication systems," IEEE Communications Magazine, vol. 52, no. 11, pp. 104-110, 2014.

[7] R. Zhang and C. K. Ho, "MIMO broadcasting for simultaneous wireless information and power transfer," IEEE Transactions on Wireless Communications, vol. 12, no. 5, pp. 1989-2001, 2013.

[8] Z. Chu, M. Johnston, and S. Le Goff, "SWIPT for wireless cooperative networks," IEEE Electronics Letters, vol. 51, no. 6, pp. 536-538, 2015.

[9] D. Yang, Q. Wu, Y. Zeng, and R. Zhang, "Energy Trade-off in Ground-to-UAV Communication via Trajectory Design," IEEE Transactions on Vehicular Technology, vol. 67, no. 07, pp. 67216726, 2018.

[10] K. Doppler, M. Rinne, C. Wijting, C. B. Ribeiro, and K. Hug, "Device-to-device communication as an underlay to LTEadvanced networks," IEEE Communications Magazine, vol. 47, no. 12, pp. 42-49, 2009.

[11] C. Yu, K. Doppler, C. B. Ribeiro, and O. Tirkkonen, "Resource sharing optimization for device-to-device communication underlaying cellular networks," IEEE Transactions on Wireless Communications, vol. 10, no. 8, pp. 2752-2763, 2011.
[12] D. Feng, L. Lu, Y.-W. Yi, G. Y. Li, G. Feng, and S. Li, "Device-todevice communications underlaying cellular networks," IEEE Transactions on Communications, vol. 61, no. 8, pp. 3541-3551, 2013.

[13] G. Fodor, E. Dahlman, G. Mildh et al., "Design aspects of network assisted device-to-device communications," IEEE Communications Magazine, vol. 50, no. 3, pp. 170-177, 2012.

[14] H. Min, W. Seo, J. Lee, S. Park, and D. Hong, "Reliability improvement using receive mode selection in the device-todevice uplink period underlaying cellular networks," IEEE Transactions on Wireless Communications, vol. 10, no. 2, pp. 413418, 2011.

[15] H. Min, J. Lee, S. Park, and D. Hong, "Capacity enhancement using an interference limited area for device-to-device uplink underlaying cellular networks," IEEE Transactions on Wireless Communications, vol. 10, no. 12, pp. 3995-4000, 2011.

[16] M. Zulhasnine, C. Huang, and A. Srinivasan, "Efficient resource allocation for device-to-device communication underlaying LTE network," in Proceedings of the 6th Annual IEEE International Conference on Wireless and Mobile Computing, Networking and Communications (WiMob '10), pp. 368-375, Ontario, Canada, October 2010.

[17] L. Fan, Z. Dong, and P. Yuan, "The Capacity of Device-toDevice Communication Underlaying Cellular Networks with Relay Links," IEEE Access, vol. 5, pp. 16840-16846, 2017.

[18] M. Seif, A. El-Keyi, K. G. Seddik, and M. Nafie, "Cooperative D2D communication in downlink cellular networks with energy harvesting capability," in Proceedings of the 13th IEEE International Wireless Communications and Mobile Computing Conference, IWCMC 2017, pp. 183-189, Spain, June 2017.

[19] V. Kaur and S. Thangjam, "A stochastic geometry analysis of RF energy harvesting based D2D communication in downlink cellular networks," in Proceedings of the 1st India International Conference on Information Processing, IICIP 2016, India, August 2016.

[20] R. I. Ansari, S. A. Hassan, and C. Chrysostomou, "A SWIPTbased device-to-device cooperative network," in Proceedings of the 2017 24th International Conference on Telecommunications (ICT), pp. 1-5, Limassol, Cyprus, May 2017.

[21] Z. Zhou, C. Gao, C. Xu, T. Chen, D. Zhang, and S. Mumtaz, "Energy-Efficient Stable Matching for Resource Allocation in Energy Harvesting-Based Device-to-Device Communications," IEEE Access, vol. 5, pp. 15184-15196, 2017.

[22] H. Meshgi, D. Zhao, and R. Zheng, "Optimal Resource Allocation in Multicast Device-to-Device Communications Underlaying LTE Networks," IEEE Transactions on Vehicular Technology, vol. 66, no. 9, pp. 8357-8371, 2017.

[23] K. Doppler, M. P. Rinne, P. Janis, C. Ribeiro, and K. Hugl, "Device-to-Device Communications; Functional Prospects for LTE-Advanced Networks," in Proceedings of the 2009 IEEE International Conference on Communications Workshops, pp. 16, Dresden, Germany, June 2009.

[24] A. Gjendemsjo, D. Gesbert, G. E. Oien, and S. G. Kiani, "Optimal power allocation and scheduling for two-cell capacity maximization," in Proceedings of the 4th International Symposium on Modeling and Optimization in Mobile, Ad Hoc and Wireless Networks (WiOpt '06), pp. 1-6, IEEE, April 2006. 


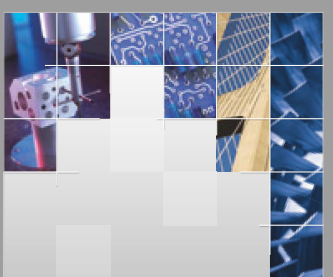

\section{Enfincering}
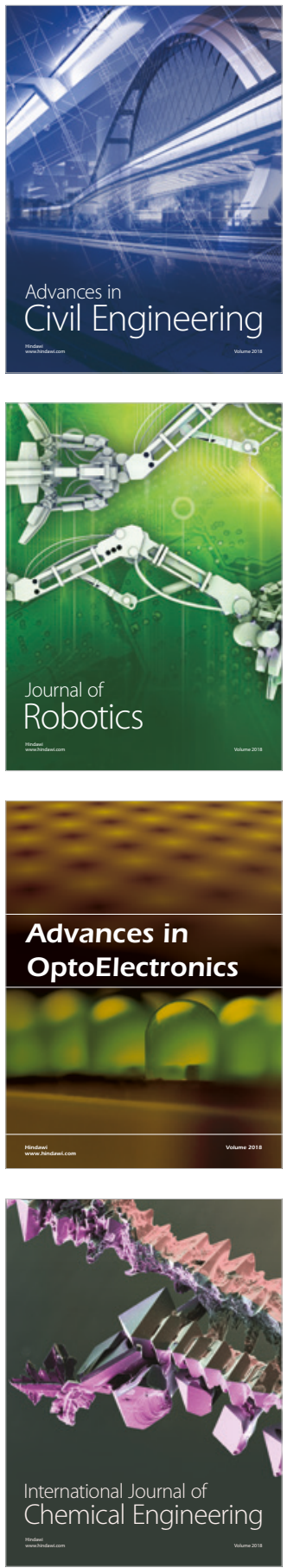

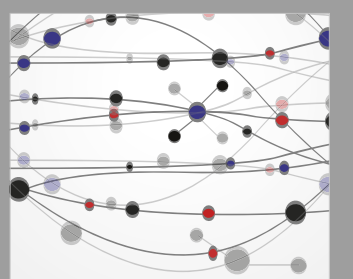

\section{Rotating \\ Machinery}

The Scientific World Journal

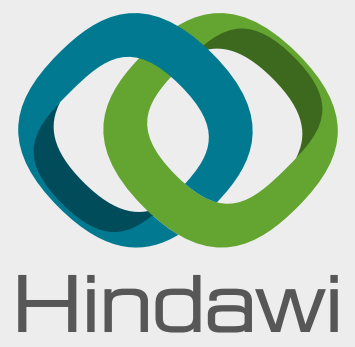

Submit your manuscripts at

www.hindawi.com
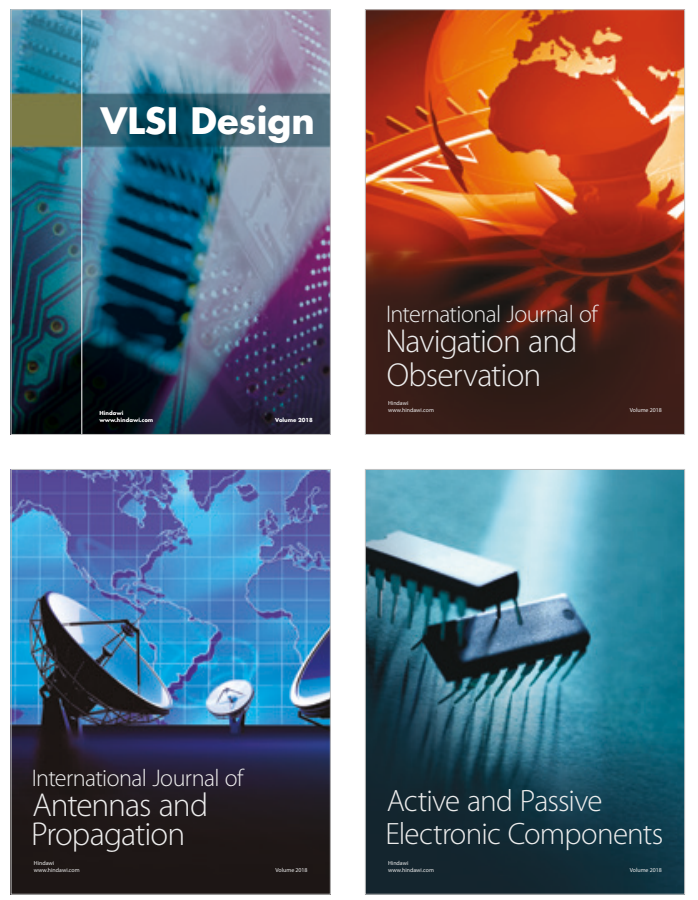
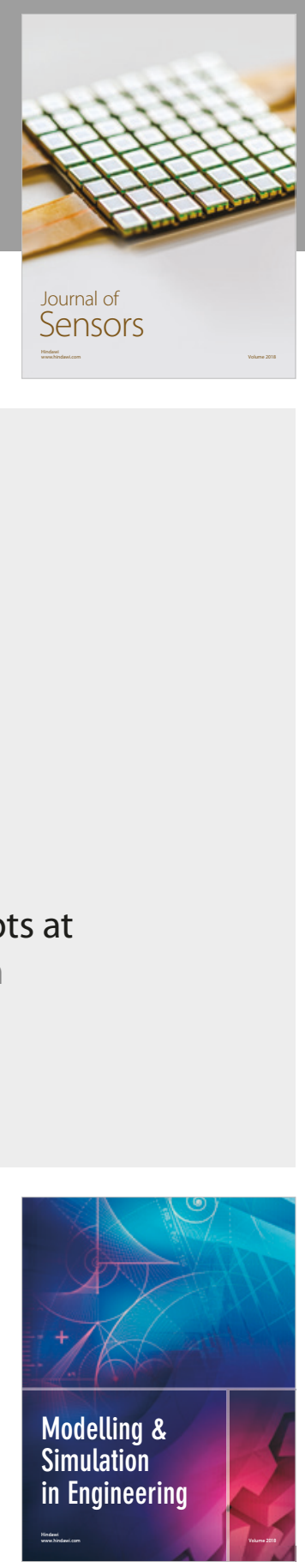

\section{Advances \\ Multimedia}
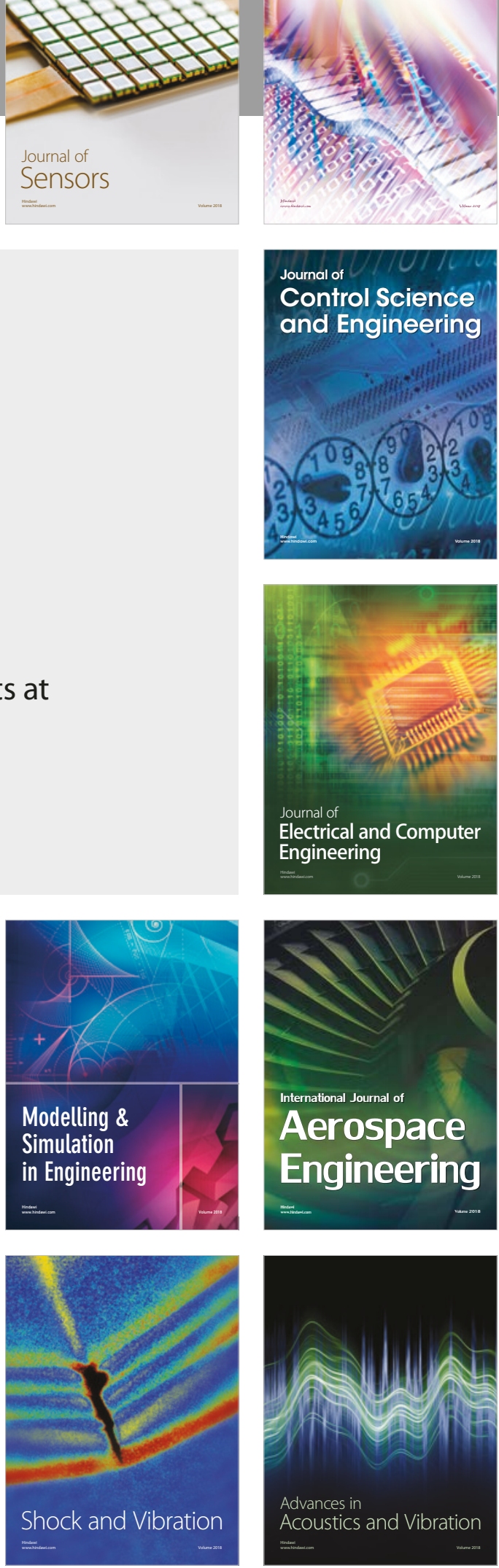